\title{
Spatial localization of the JAG1/Notch1/osteopontin cascade modulates extrahepatic metastasis in hepatocellular carcinoma
}

\author{
TONG-CHUN XUE, JING-HUAI ZOU, RONG-XIN CHEN, JIE-FENG CUI, ZHAO-YOU TANG and SHENG-LONG YE
}

\author{
Liver Cancer Institute, Zhongshan Hospital, Fudan University; Key Laboratory of Carcinogenesis \\ and Cancer Invasion (Fudan University), Ministry of Education, Shanghai, P.R. China
}

Received February 8, 2014; Accepted February 24, 2014

DOI: 10.3892/ijo.2014.2630

\begin{abstract}
The model of Notch-driven carcinogenesis and development of hepatocellular carcinoma remains controversial and is based on observations of developmental stage- and dose-dependent Notch activation. In this study, the relevance of the spatial distribution of Notch cascade members to the promotion of hepatocellular carcinoma metastasis was evaluated. The spatial expression patterns of the members of the Jagged1 (JAG1)/Notch1 cascade in HCC were evaluated in a tissue microarray of 112 tumors and 46 peri-tumors. Regulation of JAG1/Notch1 on osteopontin (OPN) was evaluated by RNA interference. Tumor cells with JAG1 expressed on the membrane (JAG1 ${ }^{\mathrm{Mem}}$ ) were more likely to undergo extrahepatic metastasis $[\mathrm{p}<0.001$; hazard ratio $(\mathrm{HR}), 0.166$; 95\% CI, 0.068-0.402], and JAG1 ${ }^{\mathrm{Mem}}$ was a strong independent prognostic factor for metastasis (HR, 0.467; 95\% CI, 0.271-0.806; $\mathrm{p}=0.006)$. JAG1 ${ }^{\mathrm{Mem}}$ also showed a strong positive correlation with Notch $1^{\mathrm{Mem}}$. In addition, tumors with JAG1 $1^{\mathrm{Mem}}$ expression had more poorly encapsulated membranes $(p=0.014)$. Furthermore, Notch $1^{\mathrm{Mem}}$ expression correlated with HCC metastasis and was the strongest predictive factor for metastasis. However, in peri-tumoral tissues, most JAG1 (45/46) and Notch1 (41/46) was localized to the cytoplasm. The expression of OPN, one of the main targets of JAG1/Notch1 signaling and a crucial metastasis-related gene in HCC, correlated significantly with JAG1 ${ }^{\mathrm{Mem}}$ expression. Knockdown of JAG1 expression or Notch1 expression induced the downregulation of OPN in HCC cells. Taken together, protein localization is a critical factor affecting the activity of the Notch cascade in the development of hepatocellular carcinoma. Furthermore, our results suggest that the JAG1/Notch1/OPN cascade represents a potential therapeutic target for hepatocellular carcinoma metastasis.
\end{abstract}

Correspondence to: Professor Sheng-Long Ye, Liver Cancer Institute, Zhongshan Hospital, Fudan University, 180 Fenglin Road, Shanghai 200032, P.R. China

E-mail: slye@shmu.edu.cn

Key words: Jagged1, Notch1, osteopontin, metastasis, hepatocellular carcinoma

\section{Introduction}

Notch signaling is involved in the tumorigenesis and progression of nearly all solid tumors (1), which makes this cascade a highly desirable therapeutic target. In particular, Notch signaling has been shown to be involved in the tumorigenesis and proliferation of hepatocellular carcinoma (HCC). However, the reports on this topic have been contradictory (2-6), and the differential expression patterns of Notch cascade members are correlated with this variability in results. In the liver, Notch acts in a developmental stage- and dose-dependent manner to coordinate biliary fate and morphogenesis (7), and similar activities have also been detected in HCC. However, temporal and concentration differences in the Notch cascade cannot completely explain the discrepancies in the Notch-related findings in $\mathrm{HCC}$, which suggests the existence of other factors that affect Notch activation in HCC.

Notch signaling is controlled at multiple levels, including through gene dosage sensitivity (8) and through cis and trans interactions between the ligand and receptor on neighboring cells or on the same cell membrane (9). Unlike most major signaling pathways, which rely on enzymatically amplified signals, Notch signaling is mediated by stoichiometric interactions between the elements of the pathway. The stoichiometric difference between receptor and ligand expression on the membrane is an important factor that affects the signaling between two interacting cells (10). Additionally, post-translational modifications introduced in the cytoplasm, such as ubiquitylation, glycosylation and phosphorylation, have emerged as key regulators of Notch signaling (11-13). Therefore, the localization of Jagged1 (JAG1) and Notch1 to the membrane or the cytoplasm directly correlates with the activity of the Notch cascade. Thus, we directed our attention to the effects of the spatial distribution of Notch signaling factors on HCC progression.

Similar to its role in organ development (14), Notch signaling impacts tumor metastasis by regulating epithelial-mesenchymal transition (EMT)-related genes (15). Breast cancer and prostate tumor models have indicated that JAG1 mediates EMT through the up-regulation of Slug and AKT signaling $(16,17)$. Recent data have further indicated that osteopontin (OPN), another EMT-related gene, is dramatically up-regulated as a result of Notch activation in HCC, suggesting that OPN is one of the main target genes of Notch 
signaling in HCC (18). Our previous research demonstrated the crucial role of OPN in HCC metastasis (19) and suggested that the JAG1-Notch1-OPN axis may play a pivotal role in HCC metastasis.

In this study, we used immunohistochemical analyses of tissue microarrays (TMAs) to evaluate whether the localization patterns of the Notch1 cascade members could affect HCC metastasis. The predictive value of the localization of JAG1-Notch1-OPN cascade members in HCC metastasis was also evaluated.

\section{Materials and methods}

Cell line. Human HCC cell lines with different metastatic potential, or MHCC $97 \mathrm{H}$ (100\% lung metatsis) and MHCC97L were established in Liver Cancer Institute of Fudan University (Shanghai, China). HCC cell line SMMC-7721 was established at Second Military Medical University (Shanghai, China); Hep3B, and HepG2 were obtained from American Type Culture Collection (Manassas, VA, USA). L02, an immortalized human liver cell line, and HCC cell lines Huh7 and HCC-7402 was obtained from Chinese Scientific Academy. The cell line was cultured in high glucose DMEM (Gibco-BRL, Grand Island, NY, USA) supplemented with 10\% fetal bovine serum (Hyclone Laboratories, Inc., Logan, UT, USA).

Patients and follow-up. One hundred twelve patients were sampled from a prospectively designed database. Ethical approval was obtained from the Zhongshan Hospital Research Ethics Committee, and informed consent was obtained from each patient. The included patients underwent hepatectomy by the same surgical team between January, 2000 and May, 2004. All of the patients had pathologically diagnosed HCC and were classified as Child-Pugh A. Routine follow-up procedures at our clinic were performed as previously described (20). Through May 2009, 56 patients were found to have lung metastasis. Ten patients with a resectable lung metastasis received a partial pneumonectomy.

TMA and immunohistochemistry. First, hematoxylin and eosin-stained slides were screened for optimal tumor content and at least $2 \mathrm{~cm}$ of tissue adjacent to the tumor (TAT). Then, the TMA was constructed according to standard procedures (21). Rabbit anti-human JAG1 (Santa Cruz Biotechnology, Santa Cruz, CA, USA) at 1:50, rabbit anti-human Notch1 (Epitomics, Burlingame, CA, USA) at 1:100 and rabbit antihuman OPN (Santa Cruz Biotechnology) at 1:200 were used as primary antibodies for detection. Detection without a primary antibody was used as a negative control.

Categories of JAG1 and Notchl expression. Two categories for JAG1 expression were established based on the distribution of immunostaining patterns: JAG1 ${ }^{\mathrm{Mem}}$ which has clear membrane expression no matter whether expression level in cytoplasm; and JAG1 ${ }^{\text {Cyto }}$ which only can be detected the cytoplasm expression). Similarly, Notch1 expression was categorized as Notch $1^{\mathrm{Mem}}$ and Notch $1^{\mathrm{Cyto}}$ based on the spatial staining pattern. Two pathologists reviewed the results independently. In addition, because OPN was only evaluated as a target of the JAG1-Notch1 cascade, we categorized OPN expression as negative or positive based on the staining intensity.

Western blot analysis. Membrane protein was extracted according to the instructions of Mem-PER Plus Membrane Protein Extraction Kit (Thermo Fisher Scientific, Rockford, IL, USA). Western blot analysis was performed according to the protocol of Bio-Rad wet transfer using the Bio-Rad Transfer Cell System (Bio-Rad, Mississauga, ON, Canada). Analyses of protein expression were performed according to the manufacturer's instructions. Rabbit anti-human JAG1 (Santa Cruz Biotechnology) 1:200, rabbit anti-human Notch1 (Epitomics) at 1:1,000, rabbit anti-human OPN (Abcam, Cambridge, MA, USA) at 1:1,000 and rabbit anti-human $\beta$-actin $\mathrm{mAb}$ (Epitomics) 1:1,000 were used as primary antibodies in detection.

RNAi. Small interference RNAs (siRNAs) to target expression of JAG1 and Notch1 were synthesized by GenePharma Corp. (Shanghai, China) The coding sequences were as follows: siJAG1-1845, 5'-GGGUCAGAAUUGUGACAUATT-3'; siJAG1-5396, 5'-GGAGUAUUCUA AUAAGCUATT-3'; siNotch1-3918, 5'-CGUCAUCAAUGGCUGCAAATT-3'; siNotch1-8240, 5'-GGAUUAAUUUGCAUCUGA ATT-3'; negative control siRNA, 5'-UUCUCCGAACGUGUCACG UTT-3'. siRNA transfection of HCCLM3 cells was performed according to the Lipofectamine 2000 protocol.

Statistical analysis. Pearson's $\chi^{2}$ test was used to compare qualitative variables in the clinical pathology analysis. When the expected sample numbers were below 5, Fisher's exact test was used. Spearman's rank test was used to detect the correlation between variables. The survival analysis included time-to-lung metastasis, overall survival (OS) and time-to-progression (TTP). The time-to-lung metastasis was calculated from the date of hepatectomy to the date of lung metastasis with a definite clinical diagnosis. The Kaplan-Meier method was used to generate the survival curves, and the log-rank test was used to compare the survival distributions between the groups. The log-rank test was also used to screen for prognostic factors in the univariate analysis. A Cox regression model was used to identify the prognostic factors related to the time-to-lung metastasis. A receiver operation curve (ROC) was used to confirm the predictive accuracy of the prognostic factors. All of the p-values were 2 -tailed, and statistical significance was set at 0.05 . The statistical analyses were completed using SPSS 18.0 software (SPSS Inc., Chicago, IL, USA).

\section{Results}

Tumor cells with JAG1 ${ }^{\text {Mem }}$ are more likely to undergo metastasis. The 112 tumors evaluated demonstrated JAG1 expression; 39 exhibited membrane expression, and 73 exhibited cytoplasmic expression. Fig. 1 shows a representative image of strong JAG1 staining in the membranes of neighboring tumor cells.

In patients with lung metastasis $(n=56), 54 \%(n=30)$ of the samples demonstrated JAG1 ${ }^{\mathrm{Mem}}$ expression, whereas most of the samples (84\%) from patients without lung metastasis $(n=56)$ demonstrated JAG1 ${ }^{\text {Cyto }}$ expression. The tumor cells 


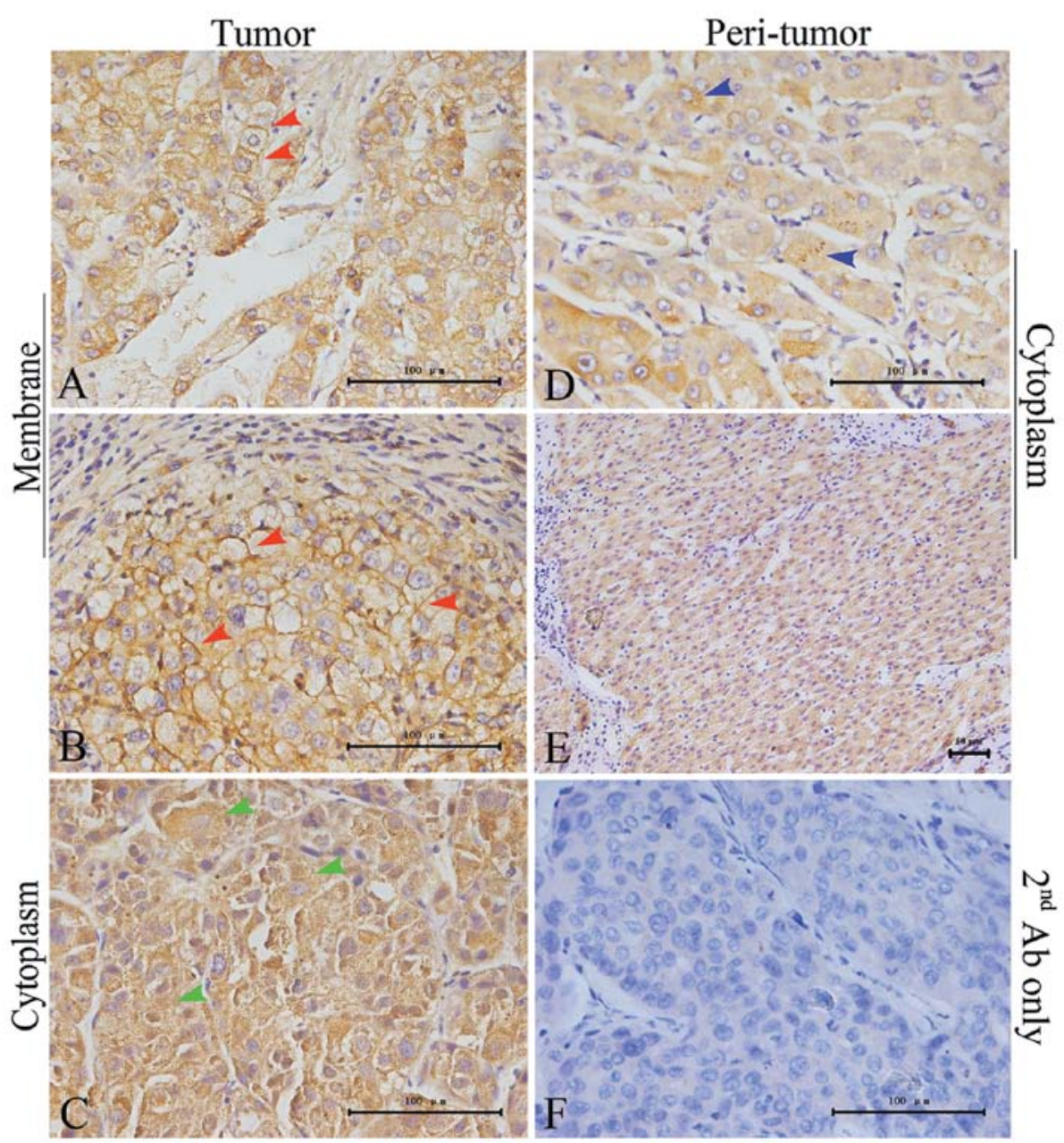

Figure 1. JAG1 staining in hepatocellular carcinoma. (A and B) Strong membrane staining was observed in the tumors. The red arrow indicates staining in neighboring tumor cells. (C) Typical cytoplasmic staining in the tumors. The green arrow indicates strong staining in the cytoplasm. (D and E) Most peri-tumors showed JAG1 ${ }^{\text {Cyto }}$ expression. Granular features (blue arrow) were typical of JAG1 ${ }^{\text {Cyto }}$ peri-tumors. (F) Samples not treated with primary antibody were used as negative controls (x400 original magnification; size bar, $100 \mu \mathrm{m}$; 200 original magnification, size bar, $50 \mu \mathrm{m}$ ).

with JAG1 ${ }^{\text {Mem }}$ frequently underwent extra-hepatic metastasis [hazard ratio (HR), 2.160; 95\% confidence interval (CI), 1.517-3.074; $\mathrm{p}<0.001]$, and $\mathrm{JAG} 1^{\mathrm{Mem}}$ correlated positively with metastasis (Spearman's rho $=0.394 ; \mathrm{p}=0.000$ ). In addition, there were no statistically significant differences in any clinicopathological variables between JAG1 ${ }^{\text {Mem }}$ and JAG1 ${ }^{\text {Cyto }}$ samples, with the exception of the tumor capsule (Table I); tumors with JAG1 ${ }^{\text {Mem }}$ had a more poorly encapsulated membrane (Spearman's rho $=0.232 ; \mathrm{p}=0.014$ ).

Patients with JAG1 ${ }^{\text {Mem }}$ tumors were also more likely to experience lung metastasis within a shorter period of time compared to those with JAG1 ${ }^{\text {Cyto }}$ tumors. In the JAG1 ${ }^{\text {Cyto }}$ group, the cumulative 1-, 3- and 5-year rates of lung metastasis-free were 73, 66 and 64\%, respectively, whereas in the JAG1 $1^{\mathrm{Mem}}$ group, the cumulative 1-, 3- and 5-year rates were 51, 31 and $22 \%$, respectively. The time-to-lung metastasis for the JAG1 ${ }^{\mathrm{Mem}}$ group was significantly shorter than that for the JAG $1^{\text {Cyto }}$ group $(\mathrm{p}<0.001$, log-rank test). The curve for time-to-lung metastasis is shown in Fig. 2A. In addition, there were significant differences in the OS and TTP between the JAG1 ${ }^{\text {Mem }}$ and JAG1 ${ }^{\text {Cyto }}$ groups (log-rank test, $\mathrm{p}=0.024$ and $\mathrm{p}=0.002$, respectively) (Fig. 3A and C).
Membrane expression of Notch1 in tumors correlates closely with metastasis. Notch1 staining was not detectable in 23 samples. In the 89 tumors with detectable Notch1 staining, 44 were Notch $1^{\mathrm{Mem}}$, and 45 were Notch $1^{\text {Cyto }}$. The typical membrane staining for Notch1 could also be observed in neighboring tumor cells (Fig. 4).

Overall, 63\% $(n=26)$ of the samples from patients with lung metastases $(n=41)$ were classified as Notch $1^{\text {Mem }}$, whereas in the patients without lung metastasis $(n=48)$, only 18 samples were Notch $1^{\mathrm{Mem}}$. Furthermore, tumors with Notch $1^{\mathrm{Mem}}$ expression were highly likely to undergo extra-hepatic metastasis (HR, 1.773, 95\% CI, 1.096-2.867; $\mathrm{p}=0.015)$, and Notch1 ${ }^{\mathrm{Mem}}$ was positively correlated with metastasis (Spearman's rho $=0.258$; $\mathrm{p}=0.015$ ). In addition, there were no statistically significant differences in any clinicopathological variables between $\mathrm{JAG1}^{\mathrm{Mem}}$ and JAG1 ${ }^{\text {Cyto }}$ (data not shown).

Patients with Notch1 ${ }^{\mathrm{Mem}}$ tumors were more likely to develop lung metastases in a shorter period of time. The time-to-lung metastasis for the Notch $1^{\mathrm{Mem}}$ group was also significantly shorter than that in the Notch $1^{\text {Cyto }}$ group (log-rank test; $\mathrm{p}=0.05)$. The time-to-lung metastasis curves are shown in Fig. 2B. In addition, there were potential differences in OS and 
Table I. Correlation between the localized expression of JAG1 and the clinical characteristics of 112 patients with hepatocellular carcinoma.

\begin{tabular}{|c|c|c|c|}
\hline \multirow[b]{2}{*}{ Variables } & \multicolumn{2}{|c|}{ No. of patients } & \multirow[b]{2}{*}{ P-value } \\
\hline & $\begin{array}{c}\mathrm{JAG}^{\mathrm{Mem}} \\
(\mathrm{n}=39)\end{array}$ & $\begin{array}{c}\text { JAG1 } 1^{\text {Cyto }} \\
(\mathrm{n}=73)\end{array}$ & \\
\hline Age, years & & & 0.067 \\
\hline$\leq 60$ & 36 & 57 & \\
\hline$>60$ & 3 & 16 & \\
\hline Gender ${ }^{\mathrm{a}}$ & & & 0.765 \\
\hline Male & 34 & 65 & \\
\hline Female & 5 & 8 & \\
\hline HBsAg & & & 0.801 \\
\hline Negative & 8 & 13 & \\
\hline Positive & 31 & 60 & \\
\hline Cirrhosis & & & 1.000 \\
\hline Absent & 31 & 57 & \\
\hline Present & 8 & 16 & \\
\hline $\operatorname{AFP}(\mu \mathrm{g} / \mathrm{l})$ & & & 0.528 \\
\hline$\leq 20$ & 11 & 26 & \\
\hline$>20$ & 28 & 47 & \\
\hline Tumor size (cm) & & & 0.539 \\
\hline$\leq 5$ & 16 & 25 & \\
\hline$>5$ & 23 & 48 & \\
\hline No. of tumor nodules & & & 0.343 \\
\hline Single & 33 & 56 & \\
\hline Multiple & 6 & 17 & \\
\hline Tumor encapsulation & & & 0.016 \\
\hline Well encapsulated & 18 & 51 & \\
\hline Poorly encapsulated & 21 & 22 & \\
\hline Microvascular invasion & & & 0.404 \\
\hline Negative & 34 & 51 & \\
\hline Positive & 15 & 22 & \\
\hline Edmondson grade & & & 0.539 \\
\hline Grade $(1 / 2)$ & 23 & 48 & \\
\hline Grade (3/4) & 16 & 25 & \\
\hline $\begin{array}{l}\text { Portal lymphatic } \\
\text { involvement }^{\mathrm{a}}\end{array}$ & & & 0.446 \\
\hline No & 35 & 69 & \\
\hline Yes & 4 & 4 & \\
\hline
\end{tabular}

${ }^{\text {a}}$ Fisher's exact test. No., number.

TTP between the Notch1 ${ }^{\mathrm{Mem}}$ and Notch $1^{\mathrm{Cyto}}$ groups (log-rank test, $\mathrm{p}=0.066$ and $\mathrm{p}=0.065$, respectively) (Fig. 3B and D).

JAGI ${ }^{\text {Mem }}$-Notchl ${ }^{\text {Mem }}$ cascade member expression is predictive of time-to-lung metastasis. To evaluate one of the most important ligand-receptor pairs in Notch signaling, we next studied the prognostic value of the localization of JAG1-Notch1
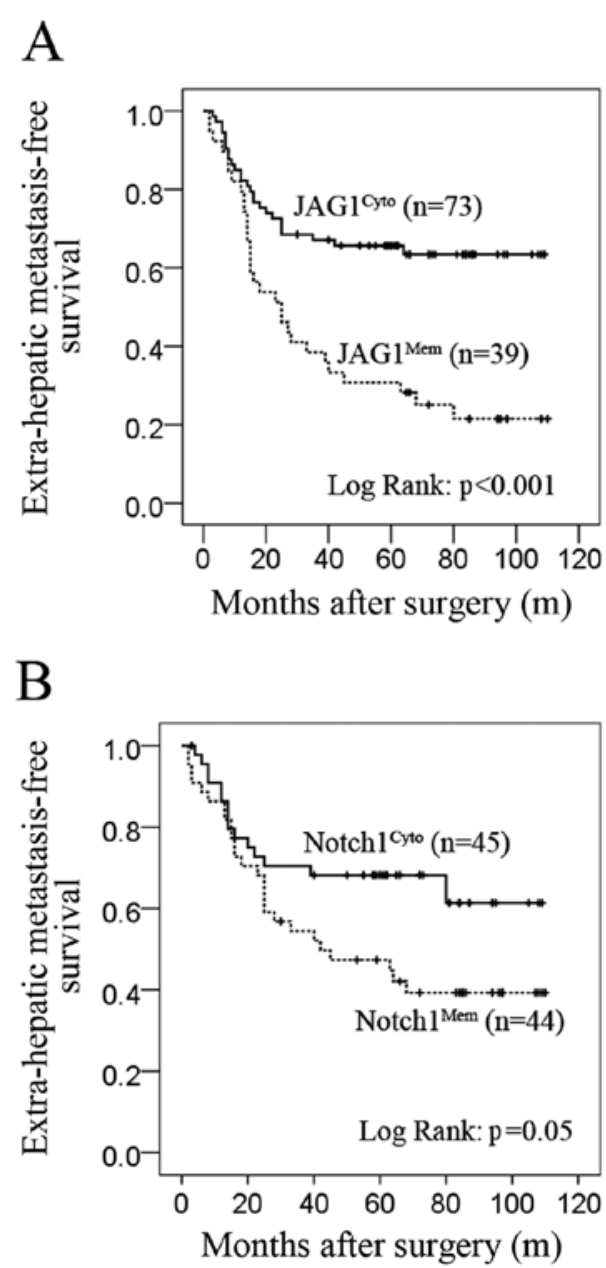

Figure 2. Membrane expression of JAG1-Notch1 correlated with a shorter time to the development of metastasis after resection. The (A) JAG1 ${ }^{\mathrm{Mem}}$ and (B) Notch $1^{\mathrm{Mem}}$ groups had a shorter time to the development of extra-hepatic metastasis compared to the JAG1 ${ }^{\text {Cyto }}$ and Notch $1^{\text {Cyto }}$ groups, respectively (Kaplan-Meier, log-rank test).

expression. The results demonstrated that $76 \%(\mathrm{n}=26)$ of JAG1 ${ }^{\mathrm{Mem}}$ tumors exhibited Notch $1^{\mathrm{Mem}}$ expression, whereas only 8 of 44 Notch $1^{\text {Cyto }}$ tumors exhibited JAG1 ${ }^{\mathrm{Mem}}$ expression. $\mathrm{JAG}^{\mathrm{Mem}}$ expression also demonstrated a strong correlation with Notch $1^{\mathrm{Mem}}$ in HCC (HR, 6.5; 95\% CI, 2.455-17.210; $\mathrm{p}<0.001$ ), and JAG1 ${ }^{\mathrm{Mem}}$ positively correlated with Notch $1^{\mathrm{Mem}}$ (Spearman's rho $=0.420 ; \mathrm{p}<0.001$ ).

Univariate analyses indicated that AFP ( $\mathrm{p}=0.013)$, microvascular invasion $(\mathrm{p}<0.001)$, portal lymphatic involvement $(\mathrm{p}=0.003)$, Edmondson grade $(\mathrm{p}=0.05), \mathrm{JAG}^{\mathrm{Mem}}(\mathrm{p}<0.001)$ and Notch $1^{\mathrm{Mem}}(\mathrm{p}=0.05)$ were potential prognostic factors for lung metastasis. Furthermore, Cox analysis showed that JAG1 ${ }^{\mathrm{Mem}}$ (HR, 0.467; 95\% CI, 0.271-0.806; $\mathrm{p}=0.006$ ), microvascular invasion (HR, 2.597; 95\% CI, 1.472-4.582; $\mathrm{p}=0.001)$ and Edmondson grade (HR, 1.791; 95\% CI, 1.045-3.070; $\mathrm{p}=0.034$ ) served as independent prognostic factors for HCC lung metastasis. In the ROC analysis, JAG1 ${ }^{\mathrm{Mem}}$ showed better specificity and sensitivity, with an AUC of 0.709 (95\% CI, 0.598-0.820; $\mathrm{p}=0.001$ ), than Notch $1^{\mathrm{Mem}}$ (AUC 0.626; 95\% CI, 0.508-0.743; $\mathrm{p}=0.043$ ) (Fig. 5A), microvascular invasion (AUC, 0.634; 95\% CI, 0.530-0.737; $\mathrm{p}=0.015$ ) or Edmondson grade (AUC, 0.598; 95\% CI, 0.493-0.704; $\mathrm{p}=0.073$ ) (Fig. 5B). 
A

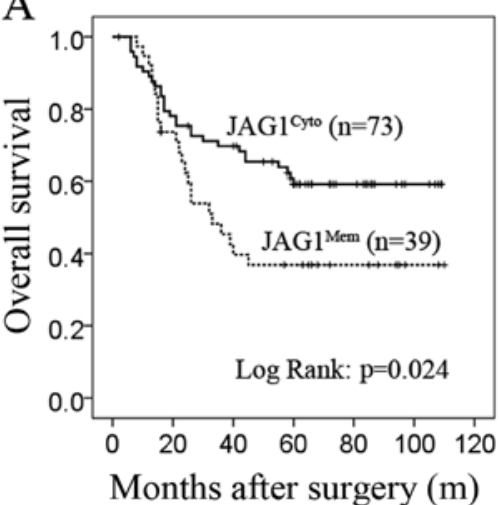

C

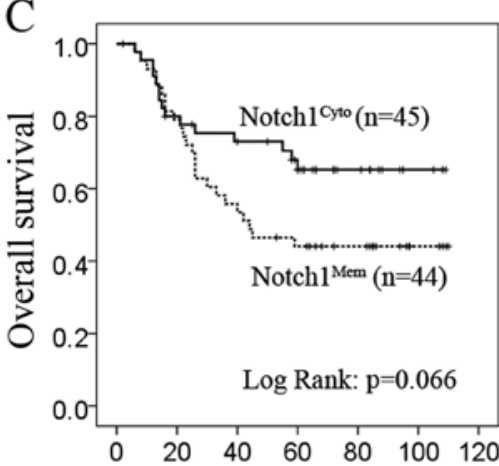

Months after surgery $(\mathrm{m})$
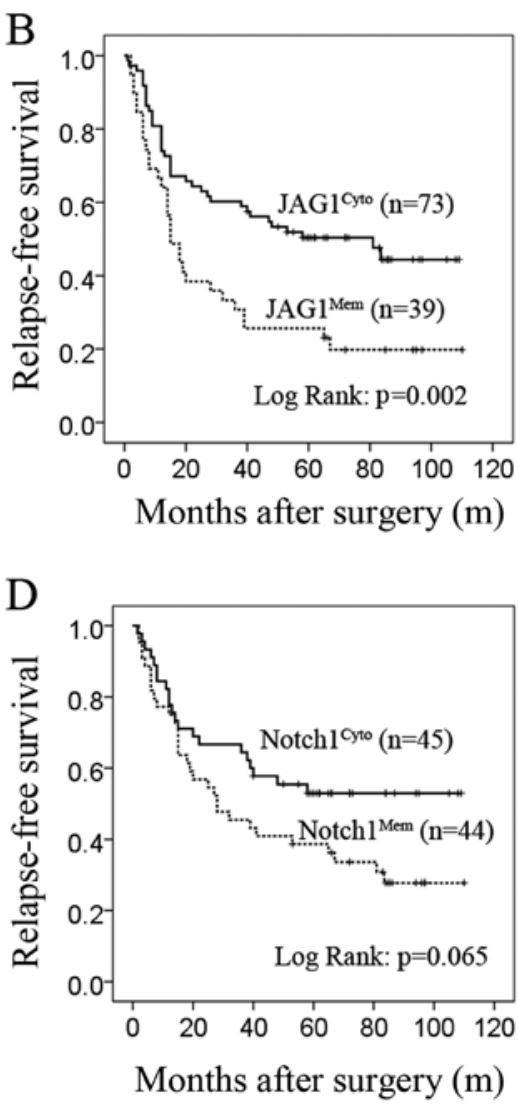

Figure 3. Membrane-type JAG1-Notch1 expression correlated with poor survival. (A) The JAG1 ${ }^{\text {Mem }}$ group demonstrated poorer overall survival than the JAG1 ${ }^{\text {Cyto }}$ group. (B) The JAG1 ${ }^{\mathrm{Mem}}$ group had a shorter time to recurrence than the JAG1 ${ }^{\text {Cyto }}$ group. (C and D) There were potential differences in overall survival and time to progression between the Notch $1^{\mathrm{Mem}}$ and Notch1 ${ }^{\text {Cyto }}$ groups (Kaplan-Meier, log-rank test).

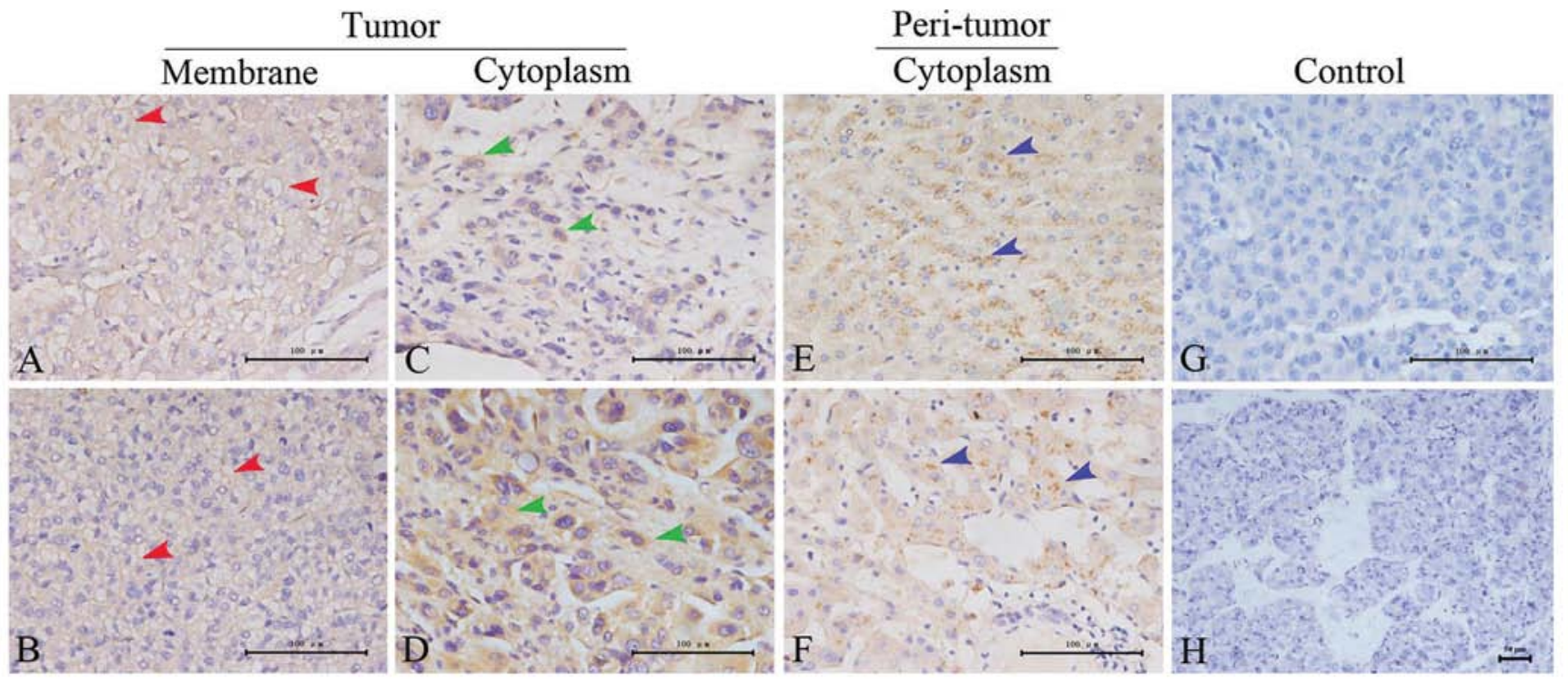

Figure 4. Notch1 staining in hepatocellular carcinoma. (A and B) Typical membrane staining for Notch1. The arrow indicates staining in neighboring tumor cells. (C and D) Cytoplasmic staining patterns were observed in the tumors. The arrow indicates strong staining in the cytoplasm. (E and F) Typical staining for Notch $1^{\text {Cyto }}$ in peri-tumor samples is shown. Cytoplasmic Notch1 expression more frequently appeared to be granular (as arrow showed). (G and H) Samples not treated with primary antibody were used as negative controls for both the tumors and peri-tumors (x400 original magnification, size bar, $100 \mu \mathrm{m}$; $\mathrm{x} 200$ original magnification, size bar, $50 \mu \mathrm{m})$.

Cytoplasmic JAG1 and Notchl expression is observed mainly in peri-tumor tissues. To evaluate the roles of JAG1-Notch1 signaling in tumors and the tumor microenvironment, we compared the spatial distributions of JAG1 and Notch1 expres- 
A

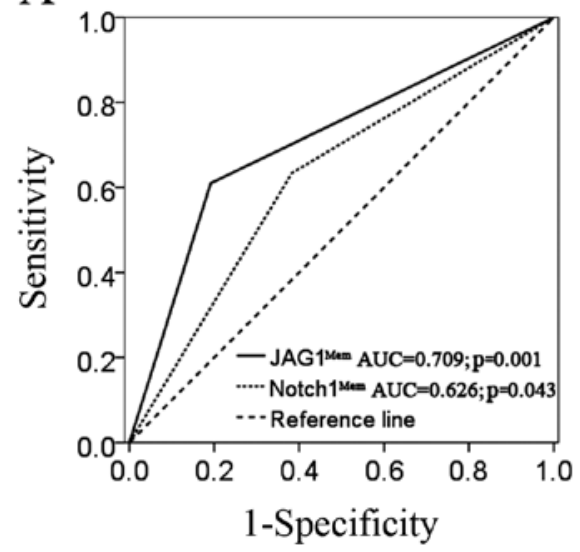

B

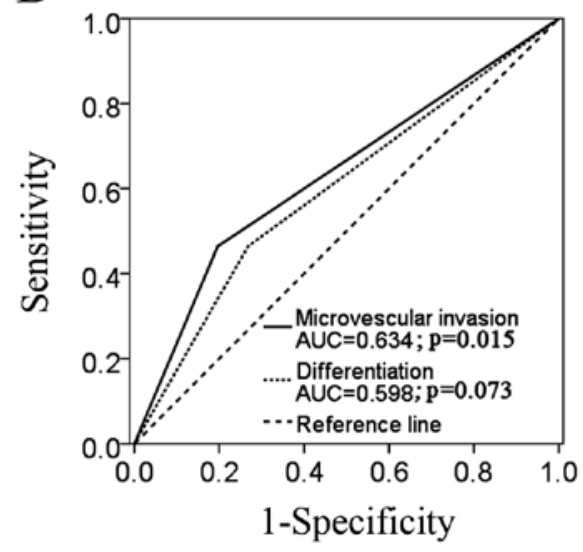

Figure 5. Receiver operation curve analysis of JAG1-Notch1 expression as a prognostic factor for metastasis. (A) The AUC values of membrane-type JAG1-Notch1 expression were $>0.05(\mathrm{P}<0.05)$, particularly for JAG1 ${ }^{\mathrm{Mem}}$, indicating great sensitivity and specificity. (B) The AUC values of microvascular invasion and differentiation were both lower than those of JAG1 ${ }^{\text {Mem }}-$ Notch $^{\text {Mem }}$.

A

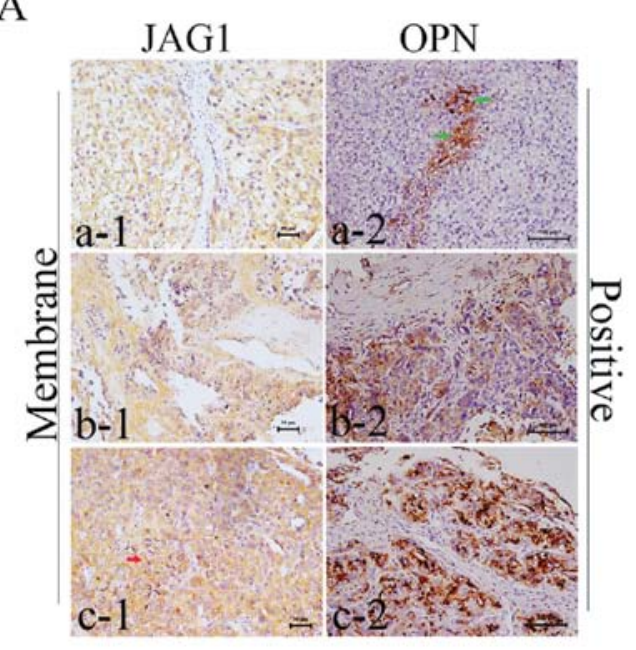

$\mathrm{C}$

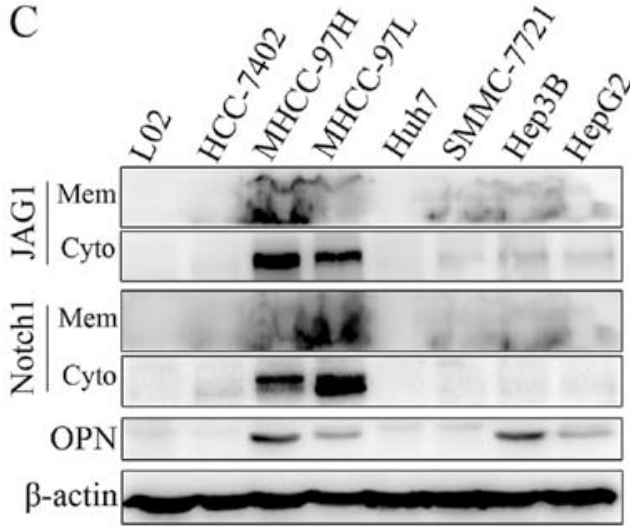

B

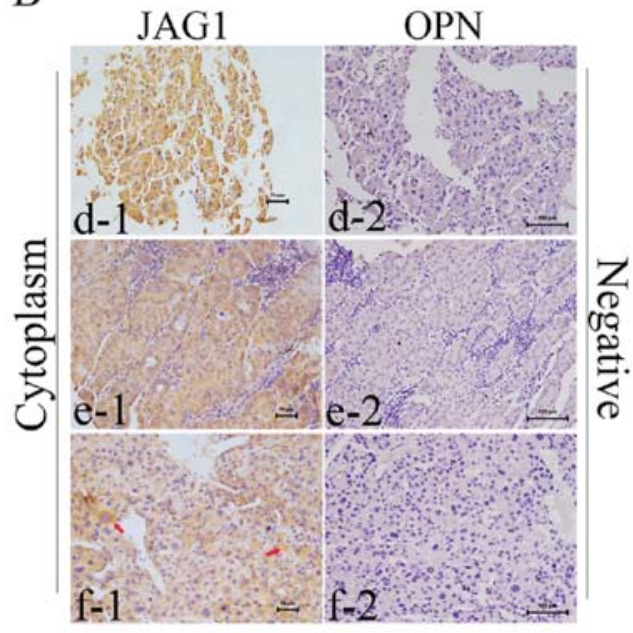

D

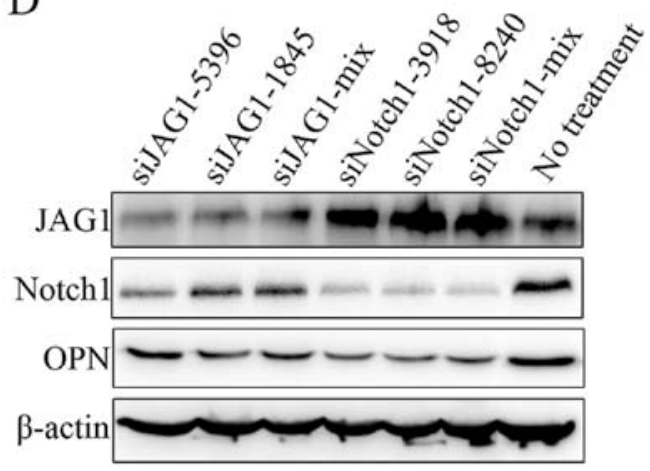

Figure 6. Membrane-type JAG1 expression correlated strongly with osteopontin staining. (A) Three cases with paired JAG1 expression on the membrane (a1-c1) and corresponding OPN staining (a2-c2) in the tissue microarray analysis. The red arrow in c-1 indicates typical membrane staining of JAG1. The green arrow indicates granular staining of OPN. (x200 original magnification). OPN, osteopontin. (B) Three cases with JAG1 expression in the cytoplasm (d1-f1) and corresponding OPN staining (d2-f2). The red arrow in $\mathrm{f}-1$ indicates the typical cytoplasmic staining pattern for JAG1. No obvious staining was detected from d-2 to f-2 (x200 original magnification). (C) Membrane and cytoplasm expression of JAG1-Notch1 in HCC cell lines. Most of HCC cell lines were showed the upregulated membrane and cytoplasm expression of JAG1 or Notch1 by western blot analysis. Highly metastatic MHCC97H had strong membrane expression of JAG1, with the consistently highly expressed OPN. (D) Knockdown of JAG1 or Notch1 expression in HCC cells by RNA interference. Downregulation of JAG1 or Notch1 decreased the OPN level in MHCC97H cells. OPN, osteopontin. 
sion in TATs, including 23 peri-tumor tissues from patients with metastasis and another 23 peri-tumor tissues from patients without metastasis. Generally, the immuno-staining for JAG1 and Notch1 in the peri-tumor tissue was weaker than that in the tumors; only one of the 46 TATs evaluated was of the JAG1 ${ }^{\mathrm{Mem}}$ type. The distribution of JAG1 ${ }^{\text {Cyto }}$ in most of the peri-tumor samples was homogenous. Small JAG1-positive granules were found in the cytoplasm (Fig. 1D). Similar to JAG1, Notch1 expression was localized to the cytoplasm in most of the peri-tumor samples (41/46). In addition, in the Notch $1^{\text {Cyto }}$ samples, the Notch1 protein typically aggregated in the cytoplasm in a granule-type pattern, which was either well distributed or regional (Fig. 4E-F).

High OPN expression correlates significantly with JAG1 ${ }^{\text {Mem }}$. Emerging reports have strongly suggested that the activation of the Notch pathway can upregulate OPN, and OPN has been shown to play critical roles in HCC metastasis. Thus, we examined the relationship between the expression of JAG1-Notch1 and that of its target gene OPN. In total, $45 \%(n=50)$ of the samples with positive JAG1 had positive OPN expression, including 19 samples with strong OPN staining. Further, 56\% $(n=22)$ of the samples in the JAG1 ${ }^{\text {Mem }}$ group $(n=39)$ had strong OPN staining, whereas only $38 \%$ of the samples in the JAG1 ${ }^{\text {Cyto }}$ group $(n=72)$ had detectable OPN expression. HCC cells with $\mathrm{JAG}^{\mathrm{Mem}}$ expression also had a strong tendency to express OPN (HR, 0.434; 95\% CI, 0.193-0.975; p=0.041), and JAG1 ${ }^{\mathrm{Mem}}$ positively correlated with OPN expression (Spearman's rho $=0.195$; $\mathrm{p}=0.042$ ). In tumor cells in which JAG1 was clearly stained on the membrane, OPN was expressed either in aggregates or uniformly (Fig. 6A), whereas in JAG1 ${ }^{\text {Cyto }}$ tumor cells, OPN was expressed relative lowly (Fig. 6B).

Knockdown of JAG1-Notchl downregulated the OPN level. The membrane expression of JAG1-Notch1 and the coincidence of expressions between JAG1-Notch1 and OPN were detected in HCC cell lines. As shown in Fig. 6C, the most of examined HCC cells had upregulated membrane expressions of both JAG1 and Notch1 when compared to normal liver cell line L02. Particularly, highly metastatic MHCC97H had strong membrane expression and cytoplasm expression of JAG1, with the consistently high expression of OPN. Further, downregulation of JAG1 expression in MHCC97H decreased OPN level. Similarly, knockdown of Notch1 induced the downregulation of OPN expression in HCC cells (Fig. 6D).

\section{Discussion}

Although a developmental stage- and dose-dependent mechanism of action for the Notch cascade was previously observed in HCC and liver tissues, the reports concerning Notchdriven carcinogenesis and HCC progression are controversial $(1,22,23)$ and suggest another possible mechanism of Notch activation. To our knowledge, this study is the first to show that the spatial distributions of Notch signaling proteins affect their roles in the development of HCC.

Notch signaling has been shown to have unique features, including gene dosage sensitivity and both cis and trans regulation mechanisms. Our findings suggest that enhanced membrane localization and expression may lead to an increased opportunity for signal communication and activation between neighboring tumor cells. In addition, strong JAG $1^{\mathrm{Mem}}$ and Notch $1^{\mathrm{Mem}}$ expression likely leads to increased trans interactions between cells and cis interactions between ligands and receptors on the same cell membrane. Additionally, because Notch activity is highly dependent on contextual cues, JAG $1^{\mathrm{Mem}}$ and Notch $1^{\mathrm{Mem}}$ expression may facilitate communication between tumor cells and the tumor microenvironment. Cytoplasmic JAG1 or Notch1 may be degraded or subject to post-translational modifications. In addition, recent evidence supports the hypothesis that the Notch-DSL pathway is bidirectional (24), suggesting that the membrane localization of JAG1-Notch1 has a unique role in Notch signaling.

In this study, the close correlation between membranelocalized JAG1-Notch1 and extra-hepatic metastasis supplied further evidence for Notch signaling in promoting HCC progression. Consistent with our findings, Notch signaling was previously shown to be involved in metastasis through an EMT mechanism in tumors and was correlated with the upregulation of E-cadherin expression in $\operatorname{HCC}(2,25)$. Furthermore, the inhibition of Notch signaling using a DAPT inhibitor was able to decrease the invasive potential of HCC (26).

To confirm the role of the JAG1-Notch1 cascade in HCC metastasis, we further evaluated the expression of the target gene OPN and the relationship between OPN and JAG1. As expected, strong OPN staining correlated closely with JAG1 $1^{\mathrm{Mem}}$ expression in the TMA analysis. Also, OPN was expressed highly in HCC cell line with strong membrane expression of JAG1. Further, knockdown of JAG1 or knockdown of Notch1 induced the downregulation of OPN expression in HCC cells. The high expression of OPN in the JAG $1^{\mathrm{Mem}}-\mathrm{Notch} 1^{\mathrm{Mem}}$ tumors further suggests the crucial role of the active JAG1-Notch1-OPN cascade in extra-hepatic metastasis. Furthermore, OPN was previously identified as a pivotal metastasis-related gene in the genomic profiling of multiple HCC pairs, suggesting that OPN is tumor specific (27). Additionally, the critical role of OPN in metastatic HCC has been well demonstrated (28). Therefore, the JAG1-Notch1-OPN cascade in HCC metastasis has tumor-specific features.

In conclusion, the spatial distribution of Notch signaling components represents another crucial factor regulating the Notch cascade in HCC. The tumor-specific JAG1-Notch1-OPN cascade was determined to be predictive of extra-hepatic HCC metastasis and poor prognosis, which suggests that this pathway may represent a therapeutic target for HCC.

\section{Acknowledgements}

This study was supported by State Key Project on Infectious Diseases of China (no. 2012ZX10002-016) and the Youth Backbone Fund from Fudan University (B-233).

\section{References}

1. Ranganathan P, Weaver KL and Capobianco AJ: Notch signalling in solid tumours: a little bit of everything but not all the time. Nat Rev Cancer 11: 338-351, 2011.

2. Zhou L, Wang DS, Li QJ, Sun W, Zhang Y and Dou KF: The down-regulation of Notch1 inhibits the invasion and migration of hepatocellular carcinoma cells by inactivating the cyclooxygenase-2/Snail/E-cadherin pathway in vitro. Dig Dis Sci 58: 1016-1025, 2013 
3. Lim SO, Park YM, Kim HS, et al: Notch1 differentially regulates oncogenesis by wildtype p53 overexpression and p53 mutation in grade III hepatocellular carcinoma. Hepatology 53: $1352-1362,2011$.

4. Liu M, Lee DF, Chen CT, et al: IKKalpha activation of NOTCH links tumorigenesis via FOXA2 suppression. Mol Cell 45: 171-184, 2012.

5. Qi R, An H, Yu Y, et al: Notch1 signaling inhibits growth of human hepatocellular carcinoma through induction of cell cycle arrest and apoptosis. Cancer Res 63: 8323-8329, 2003.

6. Viatour P, Ehmer U, Saddic LA, et al: Notch signaling inhibits hepatocellular carcinoma following inactivation of the $\mathrm{RB}$ pathway. J Exp Med 208: 1963-1976, 2011.

7. Zong Y, Panikkar A, Xu J, et al: Notch signaling controls liver development by regulating biliary differentiation. Development 136: 1727-1739, 2009.

8. Mazzone M, Selfors LM, Albeck J, et al: Dose-dependent induction of distinct phenotypic responses to Notch pathway activation in mammary epithelial cells. Proc Natl Acad Sci USA 107: 5012-5017, 2010.

9. D'Souza B, Meloty-Kapella L and Weinmaster G: Canonical and non-canonical Notch ligands. Curr Top Dev Biol 92: 73-129, 2010.

10. Artavanis-Tsakonas S and Muskavitch MA: Notch: the past, the present, and the future. Curr Top Dev Biol 92: 1-29, 2010

11. Kovall RA and Blacklow SC: Mechanistic insights into Notch receptor signaling from structural and biochemical studies. Curr Top Dev Biol 92: 31-71, 2010.

12. Le Bras S, Loyer N and Le Borgne R: The multiple facets of ubiquitination in the regulation of notch signaling pathway. Traffic 12: 149-161, 2011.

13. Fortini ME: Notch signaling: the core pathway and its posttranslational regulation. Dev Cell 16: 633-647, 2009.

14. De la Pompa JL and Epstein JA: Coordinating tissue interactions: Notch signaling in cardiac development and disease. Dev Cell 22: 244-254, 2012.

15. Wang Z, Li Y, Kong D and Sarkar FH: The role of Notch signaling pathway in epithelial-mesenchymal transition (EMT) during development and tumor aggressiveness. Curr Drug Targets 11: 745-751, 2010.

16. Leong KG, Niessen K, Kulic I, et al: Jagged1-mediated Notch activation induces epithelial-to-mesenchymal transition through Slug-induced repression of E-cadherin. J Exp Med 204: 2935-2948, 2007.
17. Santagata S, Demichelis F, Riva A, et al: JAGGED1 expression is associated with prostate cancer metastasis and recurrence. Cancer Res 64: 6854-6857, 2004.

18. Villanueva A, Alsinet C, Yanger K, et al: Notch signaling is activated in human hepatocellular carcinoma and induces tumor formation in mice. Gastroenterology 143: 1660-1669.e7, 2012.

19. Chen RX, Xia YH,Xue TC,Zhang H and Ye SL: Downregulation of osteopontin inhibits metastasis of hepatocellular carcinoma cells via a mechanism involving MMP-2 and uPA. Oncol Rep 25: 803-808, 2011

20. Xue TC, Han D, Chen RX, et al: High expression of CXCR7 combined with alpha fetoprotein in hepatocellular carcinoma correlates with extra-hepatic metastasis to lung after hepatectomy. Asian Pac J Cancer Prev 12: 657-663, 2011.

21. Simon R, Mirlacher M and Sauter G: Immunohistochemical analysis of tissue microarrays. Methods Mol Biol 664: 113-126, 2010.

22. Wang M, Xue L, Cao Q, et al: Expression of Notch1, Jagged1 and beta-catenin and their clinicopathological significance in hepatocellular carcinoma. Neoplasma 56: 533-541, 2009.

23. Lim SO, Kim HS, Quan X, et al: Notch1 binds and induces degradation of Snail in hepatocellular carcinoma. BMC Biol 9: 83, 2011. Notch signaling: emerging role and mechanism. Trends Cell Biol 22: 257-265, 2012.

25. Wang XQ, Zhang W, Lui EL, et al: Notch1-Snail1-E-cadherin pathway in metastatic hepatocellular carcinoma. Int J Cancer 131: E163-E172, 2012.

26. Zhou L, Wang DS, Li QJ, Sun W, Zhang Y and Dou KF: Downregulation of the Notch signaling pathway inhibits hepatocellular carcinoma cell invasion by inactivation of matrix metalloproteinase-2 and -9 and vascular endothelial growth factor. Oncol Rep 28: 874-882, 2012.

27. Ye QH, Qin LX, Forgues M, et al: Predicting hepatitis B virus-positive metastatic hepatocellular carcinomas using gene expression profiling and supervised machine learning. Nat Med 9: 416-423, 2003

28. Dong QZ, Zhang XF, Zhao Y, et al: Osteopontin promoter polymorphisms at locus -443 significantly affect the metastasis and prognosis of human hepatocellular carcinoma. Hepatology 57: 1024-1034, 2012. 